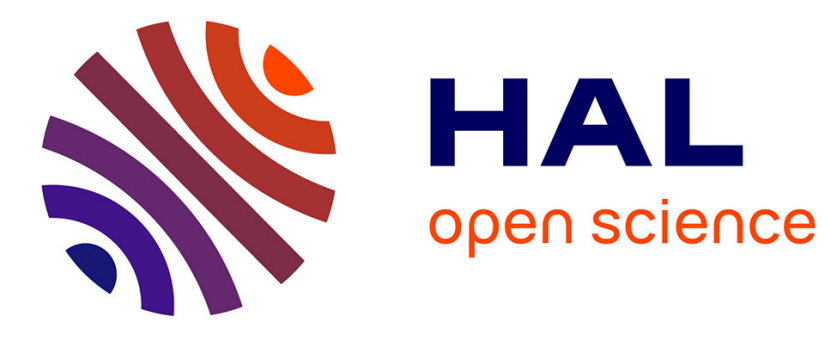

\title{
Dark solitons in a superfluid Fermi gas
}

Mauro Antezza, Franco Dalfovo, Lev P Pitaevskii, Sandro Stringari

\section{To cite this version:}

Mauro Antezza, Franco Dalfovo, Lev P Pitaevskii, Sandro Stringari. Dark solitons in a superfluid Fermi gas. Physical Review A : Atomic, molecular, and optical physics [1990-2015], 2007, 76 (4), pp.043610. 10.1103/PhysRevA.76.043610 . hal-02964061

\section{HAL Id: hal-02964061 \\ https://hal.science/hal-02964061}

Submitted on 12 Oct 2020

HAL is a multi-disciplinary open access archive for the deposit and dissemination of scientific research documents, whether they are published or not. The documents may come from teaching and research institutions in France or abroad, or from public or private research centers.
L'archive ouverte pluridisciplinaire HAL, est destinée au dépôt et à la diffusion de documents scientifiques de niveau recherche, publiés ou non, émanant des établissements d'enseignement et de recherche français ou étrangers, des laboratoires publics ou privés. 


\title{
Dark solitons in a superfluid Fermi gas
}

\author{
Mauro Antezza, ${ }^{1}$ Franco Dalfovo, ${ }^{1}$ Lev P. Pitaevskii,,${ }^{1,2}$ and Sandro Stringari ${ }^{1}$ \\ ${ }^{1}$ Dipartimento di Fisica, Università di Trento and CNR-INFM BEC, Via Sommarive 14, I-38050 Povo, Italy \\ ${ }^{2}$ Kapitza Institute for Physical Problems, ul. Kosygina 2, 119334 Moscow, Russia
}

(Received 5 June 2007; published 10 October 2007)

\begin{abstract}
We investigate the behavior of dark solitons in a superfluid Fermi gas along the BCS-BEC crossover by solving the Bogoliubov-de Gennes equations and looking for real and odd solutions for the order parameter. We show that in the resonance unitary region, where the scattering length is large, the density profile of the soliton has a deep minimum, differently from what happens in the BCS regime. The superfluid gap is found to be significantly quenched by the presence of the soliton due to the occurrence of Andreev fermionic bound states localized near the nodal plane of the order parameter.
\end{abstract}

DOI: 10.1103/PhysRevA.76.043610

PACS number(s): 03.75.Kk, 03.75.Lm, 03.75.Ss

\section{INTRODUCTION}

The interplay between effects of coherence and interaction is one of the most interesting features exhibited by superfluids. It shows up, in particular, through the occurrence of topological excitations such as vortices and solitons. These have been the object of systematic investigations in the case of ultracold Bose gases, where coherence is the result of Bose-Einstein condensation (BEC) and is associated with long-range order in the one-body density matrix. The situation is even more interesting in the case of Fermi gases where coherence originates from the interaction between particles which, at low temperature, brings the system into a superfluid phase characterized by long-range order in the two-body density matrix. For this reason, the consequences of coherence on measurable quantities, like the density distribution of the gas, are more indirect and subtle than for bosons.

Fermi superfluid gases are now available experimentally in ${ }^{40} \mathrm{~K}$ and ${ }^{6} \mathrm{Li}$ and various regimes along the BCS-BEC crossover can be explored by tuning the atomic $s$-wave scattering length $a$ through the Feshbach resonances exhibited by these atoms. When $a$ is small and negative the system is described by the BCS theory of superfluidity. Conversely when $a$ is small and positive dimers of atoms of different spin are formed and the system behaves like a BEC of molecules. Near resonance the scattering length is much larger than the average interparticle distance and the system enters the so-called unitary regime. Quantized vortices have been recently observed along the BCS-BEC crossover [1] and have been the object of several theoretical papers [2-6].

In this work we investigate another important class of nonlinear topological excitations: dark solitons. In three dimensions (3D) a dark soliton is characterized by a real order parameter which changes sign at a planar node (a point node in $1 \mathrm{D})$. In the BEC regime the soliton is a solution of the Gross-Pitaevskii (GP) equation for the order parameter of the condensate with repulsive interaction [7]. In a uniform 3D system dark solitons are known to be unstable via the snake instability, i.e., a sinusoidal transverse oscillation of the planar node. In trapped gases, however, the instability time scale can be very long, so that solitons can indeed be observed [8].
While in the BEC case the node of the order parameter causes a notch in the density distribution, in a BCS superfluid the density is almost unaffected by the presence of the node [9]. The situation is similar to the one of vortices. As in that case, the behavior of the density along the BCS-BEC crossover is expected to be interesting and rather nontrivial, as a result of the delicate balance of coherence and nonlinear interactions. A major question concerns the behavior at unitary where no exact many-body theory is presently available. The problem also shares useful analogies with the interference between two expanding Fermi superfluid gases where the order parameter is expected to exhibit an oscillating behavior with a change of sign, but no quantitative predictions are available concerning the behavior of the density.

\section{BOGOLIUBOV-de GENNES THEORY}

We investigate the problem by using a mean-field theory for a 3D Fermi gas at zero temperature, based on the solution of the Bogoliubov-de Gennes (BdG) equations $[10,11]$ :

$$
\left[\begin{array}{cc}
\hat{H} & \Delta(\mathbf{r}) \\
\Delta^{*}(\mathbf{r}) & -\hat{H}
\end{array}\right]\left[\begin{array}{l}
u_{\eta}(\mathbf{r}) \\
v_{\eta}(\mathbf{r})
\end{array}\right]=\varepsilon_{\eta}\left[\begin{array}{l}
u_{\eta}(\mathbf{r}) \\
v_{\eta}(\mathbf{r})
\end{array}\right],
$$

where $\hat{H}=-\hbar^{2} \nabla^{2} / 2 M+V_{\text {ext }}(\mathbf{r})-\mu$ is the single-particle grand-canonical Hamiltonian. For given chemical potential $\mu$ and order parameter of the superfluid phase, $\Delta(\mathbf{r})$, these equations provide the spectrum $\varepsilon_{\eta}$ and quasiparticle amplitudes $u_{\eta}(\mathbf{r})$ and $v_{\eta}(\mathbf{r})$, which are required to satisfy the normalization relation $\int d^{3} \mathbf{r}\left[u_{\eta^{\prime}}^{*}(\mathbf{r}) u_{\eta}(\mathbf{r})+v_{\eta^{\prime}}^{*}(\mathbf{r}) v_{\eta}(\mathbf{r})\right]=\delta_{\eta^{\prime} \eta^{\prime}}$. The above equations must be solved together with the equations for the order parameter and the density:

$$
\begin{gathered}
\Delta(\mathbf{r})=-g \sum_{\eta} u_{\eta}(\mathbf{r}) v_{\eta}^{*}(\mathbf{r}), \\
n(\mathbf{r})=2 \sum_{\eta}\left|v_{\eta}(\mathbf{r})\right|^{2} .
\end{gathered}
$$

This is done by means of an iterative procedure, which starts from a trial function $\Delta(\mathbf{r})$ and converges to the selfconsistent solution of Eqs. (1)-(3). All sums in the equations are limited by an energy cutoff $0 \leqslant \varepsilon_{\eta} \leqslant E_{c}$. This cutoff is 
required in order to cure the ultraviolet divergences and is accompanied by a regularization of the interaction parameter $g$ according to [11]

$$
\frac{1}{k_{F} a}=\frac{8 \pi \varepsilon_{F}}{g k_{F}^{3}}+\frac{2}{\pi} \sqrt{\frac{E_{c}}{\varepsilon_{F}}} .
$$

Here $a$ is the 3D $s$-wave scattering length characterizing the interaction between atoms of different spins, while $\varepsilon_{F}$ $=\hbar^{2} k_{F}^{2} / 2 M$ and $k_{F}=\left(3 \pi^{2} n_{0}\right)^{1 / 3}$ are the Fermi energy and momentum of a uniform ideal Fermi gas of density $n_{0}$ [12]. For sufficiently large values of $E_{c}$ the final results should not depend on the choice of the cutoff. It is worth stressing that the above equations reduce to the stationary GP equation for the order parameter of a condensate of molecules in the BEC limit [13], the interaction between dimers being, however, given by the mean-field value $2 a$ instead of the exact value $0.6 a$ [14]. Although approximate, this mean-field theory is expected to give a comprehensive and reasonably accurate picture of the BCS-BEC crossover.

\section{RESULTS FOR A DARK SOLITON}

We look for solutions of Eqs. (1)-(3) corresponding to a soliton at rest in the superfluid and in the absence of external potential $\left(V_{\mathrm{ext}}=0\right)[15]$. The order parameter $\Delta(z)$ is chosen to be a real and odd function of $z$, having a node in the $x y$ plane at $z=0$. The density $n(z)$ is an even function of $z$. The condition of reality implies the absence of currents, which are in general associated with a $z$ dependence of the phase of the order parameter. The calculation is done in a finite box of size $L \times L_{\perp}^{2}$, where $L$ is the size along $z$ and all solutions are forced to vanish at the boundaries. If the box size is large enough, the effects of the boundaries on the soliton are vanishingly small. Due to the translational symmetry in the transverse direction, the $\mathrm{BdG}$ eigenfunctions can be

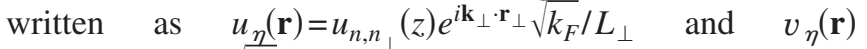

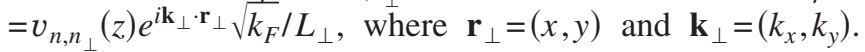
The transverse momentum is quantized according to the rule $k_{x}=2 \pi n_{x} / L_{\perp}$ and $k_{y}=2 \pi n_{y} / L_{\perp}$, with $n_{\perp}=\left(n_{x}, n_{y}\right)$ and $n_{x}, n_{y}$ integers. We consider a gas in a box with $L=40 k_{F}^{-1}$ and $L_{\perp}=20 k_{F}^{-1}$, and we also set $E_{c}=50 \varepsilon_{F}$, which turns out to be large enough for a reasonable convergence.

In Fig. 1 we show the order parameter (a) and the density (b) for a dark soliton with $\left(k_{F} a\right)^{-1}=-1,0$, and +1 . Both quantities are normalized to their asymptotic values far away from the soliton, $n_{0}=k_{F}^{3} / 3 \pi^{2}$ and $\Delta_{0}$. For $\Delta_{0}$ and $\mu$ we find the values $\Delta_{0} \simeq 0.21 \varepsilon_{F}$ and $\mu \simeq 0.96 \varepsilon_{F}$ for $\left(k_{F} a\right)^{-1}=-1$ (BCS side), $\Delta_{0} \simeq 0.70 \varepsilon_{F}$ and $\mu \simeq 0.61 \varepsilon_{F}$ for $\left(k_{F} a\right)^{-1}=0$ (unitarity), and $\Delta_{0} \simeq 1.5 \varepsilon_{F}$ and $\mu \simeq-0.84 \varepsilon_{F}$ for $\left(k_{F} a\right)^{-1}=+1$ (BEC side). These values almost coincide with those of an infinite uniform system when calculated with the same value of the cutoff energy. This proves that the size of the box is large enough to neglect its effects on the calculations. They instead differ from the values calculated in a uniform gas in the limit of infinitely large $E_{c}$. The difference is about $\simeq 10 \%$ on the BEC side and $\simeq 1 \%$ in the other cases. A larger discrepancy is of course obtained by using a lower cutoff energy, especially on the BEC side of the resonance where the formation

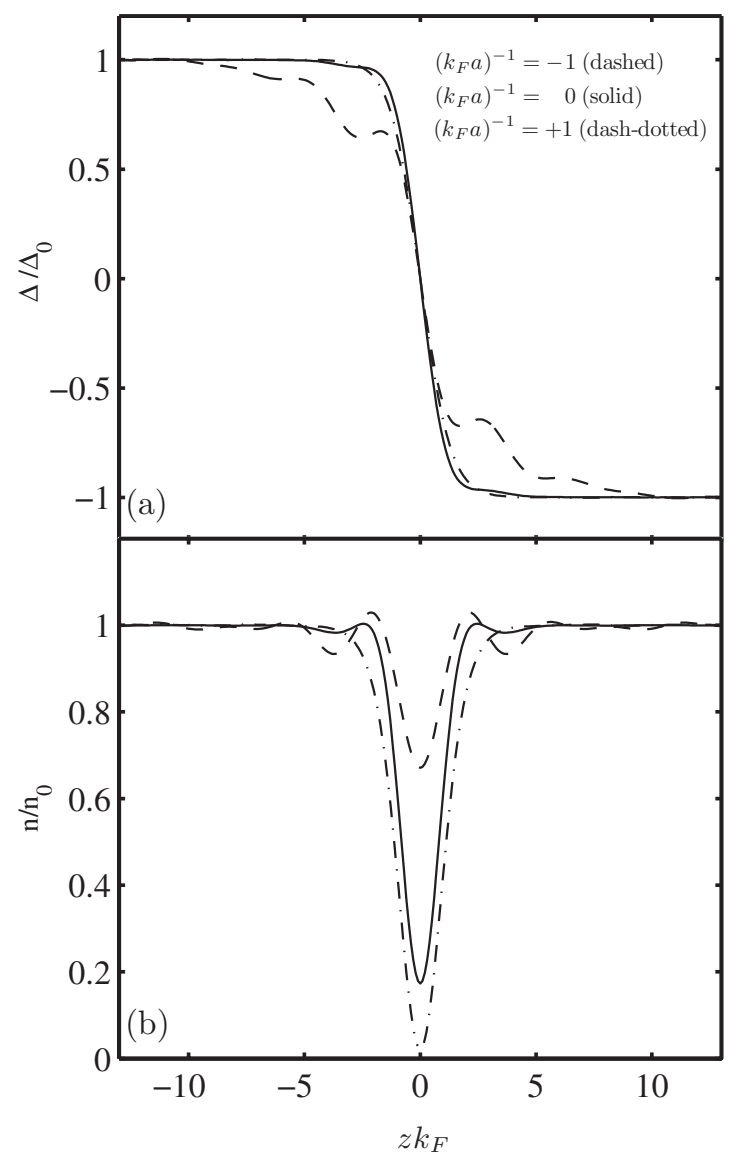

FIG. 1. (a) Order parameter and (b) density for a dark soliton with $\left(k_{F} a\right)^{-1}=0$ and \pm 1 . The value $\left(k_{F} a\right)^{-1}=0$ (solid line) corresponds to unitarity, while $\left(k_{F} a\right)^{-1}=-1$ (dashed lines) and $\left(k_{F} a\right)^{-1}=+1$ (dot-dashed lines) are on the BCS and BEC sides of the resonance, respectively. Both $\Delta(z)$ and $n(z)$ are normalized to their asymptotic values far away from the soliton.

of molecules with energy $\sim \hbar^{2} / m a^{2}$ requires large values of $E_{c}$ to reach convergence.

Figure 1(b) shows the occurrence of a deep depletion of the density at unitarity (solid line) with $a \simeq 80 \%$ contrast, comparable to the one of the BEC regime (dash-dotted line). On the BCS side (dashed line), conversely, the contrast is only $\simeq 30 \%$ at $\left(k_{F} a\right)^{-1}=-1$ and becomes exponentially small in the limit $k_{F}|a| \ll 1$. These results are consistent with those obtained for the profile of a vortex core $[5,6]$.

In Fig. 1(a) one notices that, as for the core of a vortex, the order parameter of the soliton in the BCS regime exhibits two length scales: a steep slope in a narrow region of the order of $k_{F}^{-1}$ and a smoother slope in a wider region of size $\xi_{\mathrm{BCS}}=\hbar v_{F} / \Delta_{0}$, where $\xi_{\mathrm{BCS}}$ is the coherence length of the Cooper pairs and $v_{F}=\sqrt{2 \varepsilon_{F} / M}$ is the Fermi velocity. For $\left(k_{F} a\right)^{-1}=-1$ one has $\xi_{\mathrm{BCS}} \simeq 10 k_{F}^{-1}$. We also find that the density exhibits oscillations with wave vector $\sim 2 k_{F}$. The same type of oscillations are found at the box boundaries, and we checked that their shape does not depend on either the box size or the cutoff energy. One can thus safely identify them as Friedel oscillations. Approaching unitarity the coherence length $\xi_{\mathrm{BCS}}$ decreases, eventually reducing to $k_{F}^{-1}$. 

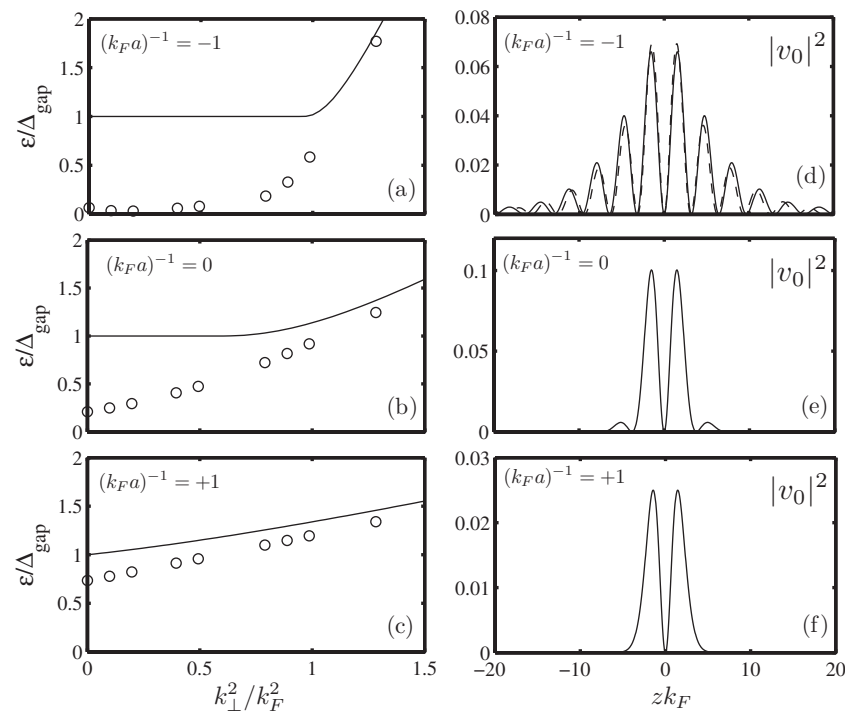

FIG. 2. Left panels: energy of the lowest single-particle states for fixed $k_{\perp}$ and for (a) $\left(k_{F} a\right)^{-1}=-1$, (b) 0 , and (c) +1 . Circles correspond to the lowest bound states in the presence of the dark soliton; solid lines correspond to the lowest-energy states $\varepsilon_{\text {bulk }}$ in a uniform superfluid (see text). Right panels: $\left|v_{0}(z)\right|^{2}$ for the lowest bound state with $k_{\perp}=0$. The dashed line in (d) corresponds to the ansatz $v_{0}(z) \sim \sin \left(k_{F} z\right) \exp \left(z / \xi_{\mathrm{BCS}}\right)$ with $\xi_{\mathrm{BCS}}=10 k_{F}^{-1}$.

Let us focus now on the single-particle excitation spectrum. In the uniform superfluid the spectrum is given by the well-known result $\varepsilon_{\text {bulk }}(k)=\left[\left(\hbar^{2} k^{2} / 2 M-\mu\right)^{2}+\Delta_{0}^{2}\right]^{1 / 2} \quad[16]$. This expression is plotted with solid lines in Figs. 2(a)-2(c) as a function of $k_{\perp}^{2}$ for the three cases $\left(k_{F} a\right)^{-1}=0$ and \pm 1 . The minimum of this function defines the gap $\Delta_{\text {gap }}$ for bulk excitations, which is $\Delta_{\text {gap }}=\Delta_{0}$ for $\mu>0$ and $\Delta_{\text {gap }}=\left[\mu^{2}+\Delta_{0}^{2}\right]^{1 / 2}$ for $\mu<0$. In the presence of the soliton, however, the eigenvalues $\varepsilon_{n, n_{\perp}}$ of the $\mathrm{BdG}$ equations exhibit a nontrivial feature: besides the continuum of bulk states with energy above $\varepsilon_{\text {bulk }}$ one finds several states even below $\varepsilon_{\text {bulk }}$. The energy of the lowest states for each $k_{\perp}$ is given in Figs. 2(a)-2(c) (open circles). The corresponding quasiparticle amplitudes are localized near the soliton, as one can see in Figs. 2(d)-2(f) where we plot the function $\left|v_{0}(z)\right|^{2}$ of the state with $k_{\perp}=0$. The origin of these localized Andreev-like states [17] resides in the fact that the energy cost for creating a fermionic excitation near a node of the order parameter is reduced with respect to the bulk value. In the BCS limit the energy of the lowest bound state, also called minigap in the context of superconductivity, is expected to be of the order of $\Delta_{0}^{2} / 2 \varepsilon_{F}$. For a vortex in a BCS superfluid described by BdG equations this result was proved in Ref. [18], but the result is rather general and can be derived also when $\Delta(z)$ is a step function $[20,21]$. In the same limit, the eigenvectors $u_{0}(z)$ and $v_{0}(z)$ of the lowest bound state behave like $\sim \cos \left(k_{F} z\right) \exp \left(z / \xi_{\mathrm{BCS}}\right)$ and $\sim \sin \left(k_{F} z\right) \exp \left(z / \xi_{\mathrm{BCS}}\right)$, respectively, as shown in Fig. $2(\mathrm{~d})$. For the minigap at $\left(k_{F} a\right)^{-1}=-1$, we find $\Delta_{\text {minigap }}$ $\simeq 0.64 \Delta_{0}^{2} / 2 \varepsilon_{F}$.

We now discuss the BEC limit, where the density profile should approach the analytic result obtained by solving the GP equation for a gas of bosons with mass $m_{B}=2 M$ interact-

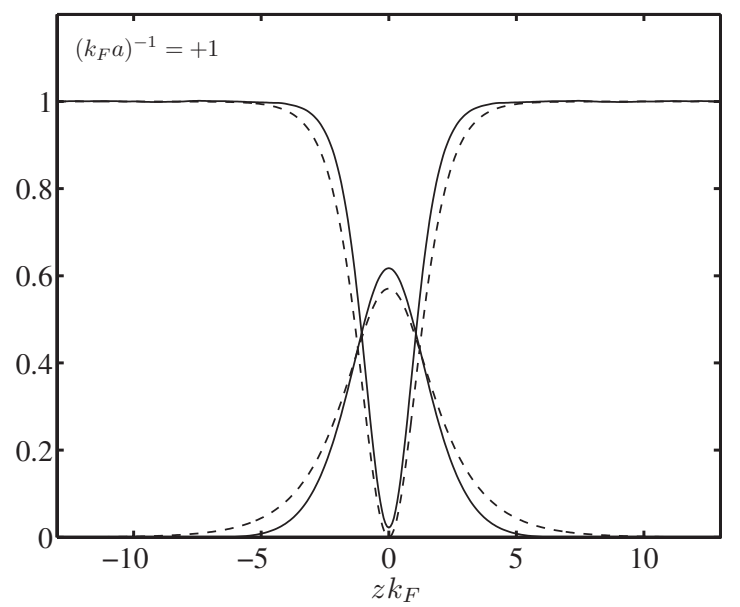

FIG. 3. Dashed lines: density profile $n_{B}(z) / n_{B 0}$ and wave function of the lowest fermionic bound state, $\Psi_{0}(z)$, in the BEC limit. Solid lines: density profile $n(z) / n_{0}$ and lowest eigenvector $u_{0}(z)$ of the BdG equations for $\left(k_{F} a\right)^{-1}=+1$.

ing with a scattering length $a_{B B}$. The density of the bosonic dimers, $n_{B}=n / 2$, is given by [7] $n_{B}(z)=n_{B 0} \tanh ^{2}\left(z / \sqrt{2} \xi_{\mathrm{BEC}}\right)$, with $n_{B 0}=n_{0} / 2=k_{F}^{3} /\left(6 \pi^{2}\right), \quad \xi_{\mathrm{BEC}}=\hbar / \sqrt{2 m_{B} g_{B B} n_{B 0}}$, and $g_{B B}=4 \pi \hbar^{2} a_{B B} / m_{B}$. Analytic solutions can be found, in the same limit, also for the fermionic bound states. In fact, these states can be obtained by solving a Schrödinger equation for a fermionic impurity of mass $m_{F}=M$ in an inhomogeneous bosonic superfluid. By minimizing the total energy functional one derives the following equation for the impurity wave function $\Psi$ :

$$
\left[-\frac{\hbar^{2}}{2 m_{F}} \partial_{z}^{2}+g_{B F} n_{B}(z)\right] \Psi(z)=\varepsilon \Psi(z),
$$

where $g_{B F}=2 \pi \hbar^{2} a_{B F} / m_{r}$ is the dimer-atom coupling constants, while $m_{r}=m_{B} m_{F} /\left(m_{B}+m_{F}\right)=2 M / 3$ is the reduced mass. The exact values of the $a_{B B}$ and $a_{B F}$ scattering lengths are equal to $0.6 a[14]$ and $1.2 a$ [22], respectively, while the BCS mean-field theory yields the Born approximation values $2 a$ and 8/3a [23]. Equation (5) corresponds to the Schrödinger equation for a particle in a modified PöschlTeller potential. The solutions are analytic [24] and include bound states. The energy of these bound states can be used to calculate the minigap for fermionic excitations in the BEC regime. In fact, the breaking of a pair causes the unbound motion of two fermions which will occupy the single-particle states $\Psi_{0}$ with the lowest energy. Using the mean-field values for the scattering length, the energy of the lowest state turns out to be exactly one-half of the bulk value $g_{B F} n_{0} / 2$ [25] so that

$$
\Delta_{\text {gap }}-\Delta_{\text {minigap }}=4 \pi \hbar^{2} a n_{0} / M .
$$

Equation (6) provides a good quantitative estimate for the gap even for $\left(k_{F} a\right)^{-1}=+1$ where the above expression gives the value $\Delta_{\text {gap }}-\Delta_{\text {minigap }}=0.26 \Delta_{\text {gap }}$ to be compared with our numerical result $\simeq 0.27 \Delta_{\text {gap }}$. In Fig. 3 the analytic curve for the density $n_{B}(z)$ and the bound-state wave function $\Psi_{0}(z)$ (dashed lines) are compared with $n(z) / 2$ and $u_{0}(z)$ obtained 
from BdG equations (solid lines). The agreement is rather good and the small differences are due to the fact that $\left(k_{F} a\right)^{-1}=+1$ is still relatively far from the true BEC limit and to the use of a finite cutoff energy $E_{c}$.

\section{CONCLUSIONS}

In conclusion, we have theoretically investigated the behavior of dark solitons in a Fermi superfluid in the BCS-BEC crossover, showing that the soliton at unitarity has a large density contrast. We have also discussed the existence of bound states. Fermionic bound states have already been the object of investigations in the context of 1D dark solitons in conducting polymers [26]. They play an important role also in the physics of domain walls in the Fulde-Ferrell-LarkinOvchinnikov states (see, for instance, Refs. [19,20] and references therein) and in the case of Josephson currents through a potential barrier [27]. In the opposite BEC limit, the binding of the unpaired Fermi atoms on the solitonic plane shares an interesting analogy with the Andreev state of
${ }^{3} \mathrm{He}$ atoms on the free surface of superfluid ${ }^{3} \mathrm{He}$ [28]. In the geometry of the soliton, the fermionic bound state can give rise to a $2 \mathrm{D}$ Fermi gas embedded into a molecular Bose superfluid. This can be particularly interesting when the molecular BEC is made starting from a slightly imbalanced spin population. The residual gas of unpaired atoms can easily fill the available states in the soliton, thus forming a polarized 2D Fermi gas. From the experimental viewpoint, it seems quite possible to observe solitons in the BCS-BEC crossover, for instance, by producing them in the molecular BEC phase with known techniques [8] and then tuning the scattering length across the Feshbach resonance, similarly to what has already been done with vortices. A natural extension of this work is the study of solitons characterized by a complex order parameter and moving in the superfluid (gray solitons).

\section{ACKNOWLEDGMENTS}

We thank S. Giorgini, E. J. Mueller, and M. Randeria for stimulating discussions. We acknowledge support by the Ministero dell’Università e della Ricerca (MiUR).
[1] M. W. Zwierlein et al., Nature (London) 435, 1047 (2005).

[2] N. Nygaard, G. M. Bruun, C. W. Clark, and D. L. Feder, Phys. Rev. Lett. 90, 210402 (2003).

[3] A. Bulgac and Y. Yu, Phys. Rev. Lett. 91, 190404 (2003).

[4] M. Machida and T. Koyama, Phys. Rev. Lett. 94, 140401 (2005).

[5] R. Sensarma, M. Randeria, and T.-L. Ho, Phys. Rev. Lett. 96, 090403 (2006).

[6] C.-C. Chien, Y. He, Q. Chen, and K. Levin, Phys. Rev. A 73, 041603(R) (2006).

[7] T. Tsuzuki, J. Low Temp. Phys. 4, 441 (1971).

[8] S. Burger, K. Bongs, S. Dettmer, W. Ertmer, K. Sengstock, A. Sanpera, G. V. Shlyapnikov, and M. Lewenstein, Phys. Rev. Lett. 83, 5198 (1999); J. Denschlag et al., Science 287, 97 (2000); B. P. Anderson, P. C. Haljan, C. A. Regal, D. L. Feder, L. A. Collins, C. W. Clark, and E. A. Cornell, Phys. Rev. Lett. 86, 2926 (2001); Z. Dutton et al., Science 293, 663 (2001); N. S. Ginsberg, J. Brand, and L. V. Hau, Phys. Rev. Lett. 94, 040403 (2005); P. Engels and C. Atherton, e-print arXiv:0704.2427; J. Brand, L. D. Carr, and B. P. Anderson, e-print arXiv:0705.1341.

[9] J. Dziarmaga and K. Sacha, Laser Phys. 15, 674 (2005).

[10] P. G. de Gennes, Superconductivity of Metals and Alloys (Benjamin, New York, 1966).

[11] A. J. Leggett, in Modern Trends in the Theory of Condensed Matter, edited by A. Pekalski and R. Przystawa (SpringerVerlag, Berlin, 1980); M. Randeria, in Bose Einstein Condensation, edited by A. Griffin, D. Snoke, and S. Stringari (Cambridge University Press, Cambridge, England, 1995).

[12] In typical experiments with ultracold gases $n_{0}$ $\sim 10^{11}-10^{12}$ atoms $/ \mathrm{cm}^{3}$ corresponding to $k_{F}^{-1}$ of few $\mu \mathrm{m}$.

[13] P. Pieri and G. C. Strinati, Phys. Rev. Lett. 91, 030401 (2003).
[14] D. S. Petrov, C. Salomon, and G. V. Shlyapnikov, Phys. Rev. Lett. 93, 090404 (2004).

[15] The existence of nonuniform solutions, such as solitons and vortices, even in the absence of an external potential, is a consequence of the nonlinear character of the self-consistent equations (1)-(3).

[16] We remind here that BdG equations (1)-(3) account for fermionic single-particle excitations and do not include bosonic collective excitations.

[17] A. F. Andreev, Zh. Eksp. Teor. Fiz. 46, 1823 (1964) [Sov. Phys. JETP 19, 1228 (1964)]; Zh. Eksp. Teor. Fiz. 49, 655 (1965); 22, 455 (1966).

[18] C. Caroli, P. de Gennes, and J. Matricon, Phys. Lett. 9, 307 (1964).

[19] T. Mizushima, K. Machida, and M. Ichioka, Phys. Rev. Lett. 94, 060404 (2005).

[20] N. Yoshida and S.-K. Yip, Phys. Rev. A 75, 063601 (2007).

[21] E. J. Mueller (private communication).

[22] G. V. Skorniakov and K. A. Ter-Martirosian, Sov. Phys. JETP 4, 648 (1957).

[23] P. Pieri and G. C. Strinati, Phys. Rev. Lett. 96, 150404 (2006).

[24] S. Flügge, Practical Quantum Mechanics (Springer-Verlag, Berlin, 1994).

[25] By using the exact values $a_{B B}=0.6 a$ and $a_{B F}=1.2 a$ the ratio between the lowest bound state and bulk state energies is 0.43 instead of 0.5 , and a second weakly bound state appears with a ratio 0.97 .

[26] A. J. Heeger et al., Rev. Mod. Phys. 60, 781 (1988).

[27] A. Spuntarelli, P. Pieri, and G. C. Strinati, Phys. Rev. Lett. 99, 040401 (2007).

[28] A. F. Andreev, Zh. Eksp. Teor. Fiz. 50, 1415 (1966) [Sov. Phys. JETP 23, 939 (1966)]. 